\title{
Identification and Automatic ICA-PSO Two-Class Classification of Time Series RSN in Shaky Hand Syndrome
}

\author{
S. P. Thaiyalnayaki, 0. Uma Maheswari \\ Department of Electronics and Communication Engineering, CEG, Anna University, Chennai, India \\ Email: sakthistructrichy@gmail.com
}

Received 5 April 2016; accepted 25 April 2016; published 22 June 2016

Copyright (C) 2016 by authors and Scientific Research Publishing Inc.

This work is licensed under the Creative Commons Attribution International License (CC BY).

http://creativecommons.org/licenses/by/4.0/

(c) (i) Open Access

\begin{abstract}
Neuro-imaging techniques are used to extract and assess brain enactments. As brain activations are free to advance in an eccentric way, data driven methods are exploited for functional localization. Three motor imbalance subjects whose scan size were $128 \times 128 \times 23$ and an aggregate of 110 volumes joined by three scans for nine acquisitions, who are on a normal age of 65 years and a set of 10 subject's simulated data was subjected to examination. To fully exploit the potential, advanced signal processing methods are applied on acquired resting state functional MRI (rsfMRI) and SimTB simulated rsfMRI. An algorithm called Independent Component Analysis-Particle Swam Optimization two-class classifier for decision support is implemented. The algorithm pre-process each simulated and real time rsfMRI scans, extract independent components(IC) from smoothed output, select eigen vector for optimized minimum misclassification from both time series data and perform 2-class classification using k-means clustering. The proposed algorithm aided the classification of about $87.5 \%$ of the functional localization of shaky hand subjects of acquired rsfMRI data. The number of highly activated voxels in the sensory motor network is more in shaky hand subjects.
\end{abstract}

Keywords

Simulated RsfMRI, Sensory Motor Network, k-Means ICA-PSO

\section{Introduction}

The multivariate methods for fMRI analysis are to assess the activity of each voxel upon activation. Hypothesis driven methods, like the standard GLM, attempt to fit data to hypotheses [1]. A method of analyzing fMRI data 
based on the statistical method of ICA allows the extraction of both transient and consistently task-related, physiologically-relevant non task-related and various artifactual components of the observed fMRI signals [1]-[3]. In addition, it has been reported that low-frequency oscillations are in the range of $0.1 \mathrm{~Hz}$ or lower contributed most to the temporal structure of the auditory, visual and sensory motor functional connectivity maps [4]. Many explored to improve activity detection, address the inter-subject variability and interpret activation maps. The prior information constrains one or more specific components to be close to a paradigm derived time course and improves the robustness of ICA in the presence of noise [4] [5].

To localize the spatial map and time courses, ten Healthy subject's rest fMRI data and three Shaky hand subjects rest data, 9 scans were employed for comparisons with simulated rest fMRI of healthy controls (HC) and shaky hand subjects. To evaluate the respective accuracies on estimated time courses and spatial layouts from the acquired rest fMRI and the simulated rsfMRI, the implementation is performed using MATLAB and various other toolboxes, SimTB, SPM, and ICA.

The paper is organized as shown. SimTB simulation, description of ICA followed by particle swarm optimization and ICA-PSO two-class classification are described in Section 2. Section 3 gives the experimental results while Section 4 provides conclusion and scope.

\section{Theoretical Description}

\subsection{Independent Component Analysis}

The BOLD time-series were arranged as a matrix Y and Independent component maps were obtained for all the acquisitions. Activations that are unanticipated were more likely to be uncovered by exploratory analysis such as ICA [6] [7]. Gaussian Kernal, 6 mm FWHM smoothing was performed. Once the components were generated using Informax ICA, the voxel values of the spatial map's Z scores were found, the threshold value for activated voxels was fixed and the spatial activation map of each component was displayed as in Figure 1. These were obtained using MATLAB Group ICA.

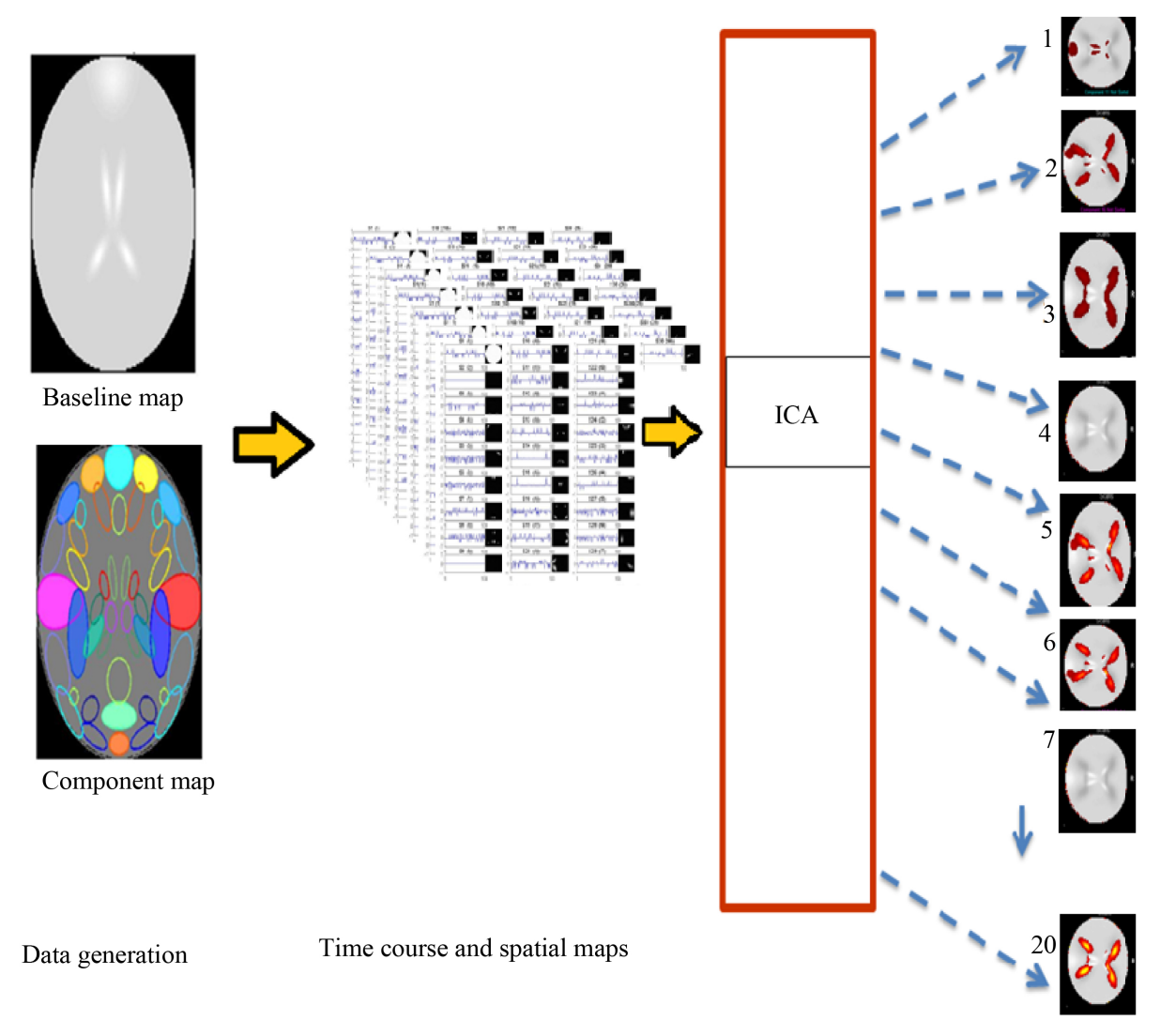

Figure 1. ICA obtained from simulated rsfMRI. 


\subsection{Particle Swarm Optimization}

To find best solution out of many in hand, bio inspired Particle swarm optimization (PSO) is implemented to identify the number of eigen values providing minimum misclassification. To Initialize PSO, the size of the swarm and the max step is chosen as 5 and 3 respectively. Search range is chosen as 10, because the iterations above that deviates from manual calculation. Initial population is evaluated by randomly initializing current position with velocity update [8]. For 3 iterations, the initializations are done as in equations shown.

$$
\begin{gathered}
\text { currentfitness }=\text { function }(\text { currentposition }) \\
\text { localbest }=\text { currentfitness } \\
\text { globalbest }=\max (\text { localbest }) \\
v=v+c 1 *(r 1 *(\text { localbest }- \text { currentposition })) \\
+c 2 *(r 2 *(\text { globalbest }- \text { currentposition })) \\
\text { currentposition }=\text { currentposition }+ \text { velocity }
\end{gathered}
$$

All swarms are evaluated and global best position is considered as the number of eigenvalues for minimum misclassification for both simulated rsfMRI and acquired rsfMRI.

\subsection{ICA-PSO Two-Class Classifier}

All meaningful independent components of simulated rsfMRI and acquired rsfMRI are utilized for classification using ICA-PSO, two-class classifier. As shown in Figure 2, five levels are constructed for the implementation of the proposed ICA-PSO, 2 class classifier. In level one, the 10 healthy controls scans and 9 abnormal subject rest fMRI scans are preprocessed to obtain normalized, Smoothed scans in nii data format. In level two, 30 independent components are obtained as 30 spatial maps using group ICA. Also 30 time series of each 19 volumes are obtained. To maintain a strategic distance from vast prominence, 30 Independent Components (ICs) are taken for classification. Level three provides highest 18 eigen vectors from the array of time series as cell data from PSO and the corresponding eigen independent components, in level four, 2 class $\mathrm{k}$ means clustering is utilized on simulated 19 shaky hand subjects and $\mathrm{HC}$ along with 19 rsfMRI scans of shaky hand subjects and $\mathrm{HC}$, to design a clinical classifier.

\section{Experimental Results}

\subsection{RsfMRI}

National Institute of Mental Health and Neuro Sciences (NIMHANS), Bangalore, INDIA has provided the required rsfMRI shakyhand and HC data. The rest fMRI of three subjects analyzed, had scan size $128 \times 128 \times 23$. A total of 110 volumes for each 3 men, 9 scans, mean age $=67 \pm 3 y$, scanned on a 3T MR scanner using a gradient echo-planar sequence of axial slice orientation of 23 slices, $\mathrm{TE}=40 \mathrm{~ms}, \mathrm{TR}=2460 \mathrm{~ms}$, flipangle $=90^{\circ}$, voxel-size $=1.8 \times 1.8 \times 6 \mathrm{~mm}$ are analyzed for activated voxel regions. The rest fMRI data is preprocessed in SPM [9] [10], localized using ICA [11] and classified using ICA-PSO, two class classifier.

\subsection{Simulated RsfMRI}

SimTB toolbox in MATLAB is utilized to obtain 19 RsfMRI simulated HC scan and shaky hand rest data. Default Mode Network, sensory network are shown in Figure 3. Twenty components of 3096 frames are obtained in SimTB for each subject. A correlation is made between ICA spatial component maps connected with these components to those created by SimTB and found that ICA delivers considerably comparable actuation maps.

\subsection{Statistical Parametric Map}

Slice time correction was performed to make the data in each slice correspond to same point in time. Spatial realignment is done, to correct changes in signal intensity over time that can arise from head motion during the scanning. The maps were then spatially normalized to a standard Talairach space and re-sampled to respective 

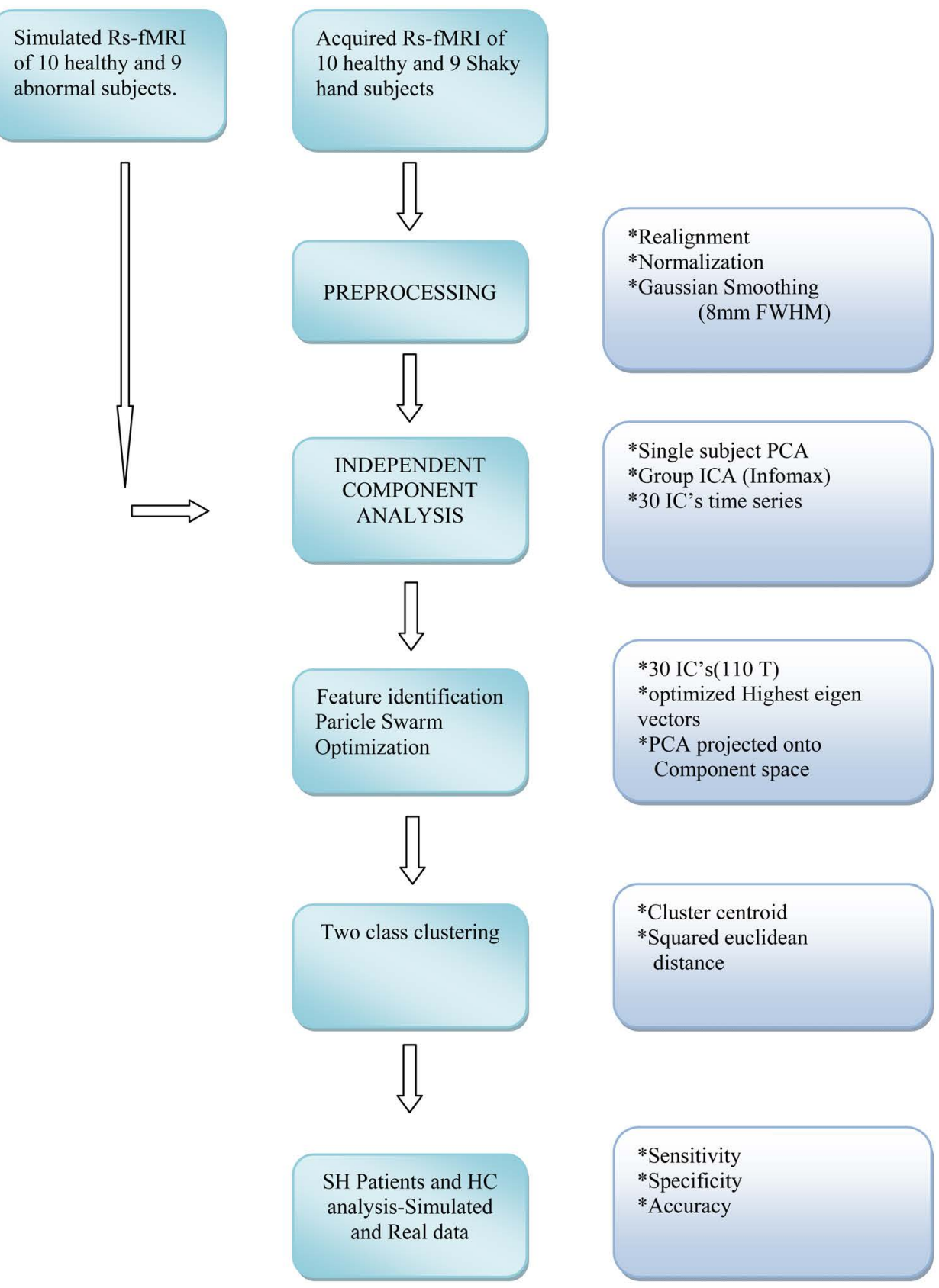

Figure 2. Proposed ICA-PSO, two-class classifier.

voxels [11]-[13]. The image volumes were convolved with Gaussian kernel to suppress noise. The SPM preprocessed output in Analyze format is given as input to gift ICA for different time series and spatial maps.

\subsection{Rest fMRI Activations}

The rest fMRI spatial maps for the recognized Independent component, Default mode network is shown in Figure 4, identified in all Healthy and abnormal subjects [11] [14].

R Executive control network is obtained as in Figure 5. This may be due to the subjects' acquisition of information through hands [15], noise observance and low level of anxiety and depression.

The sensory motor network of subject 3 with shaky hands as against the healthy controls (HC) was shown in Figure 6. Rest set fMRI Acquisition of Subject 3 shows varied activation regions due to non silence observance 

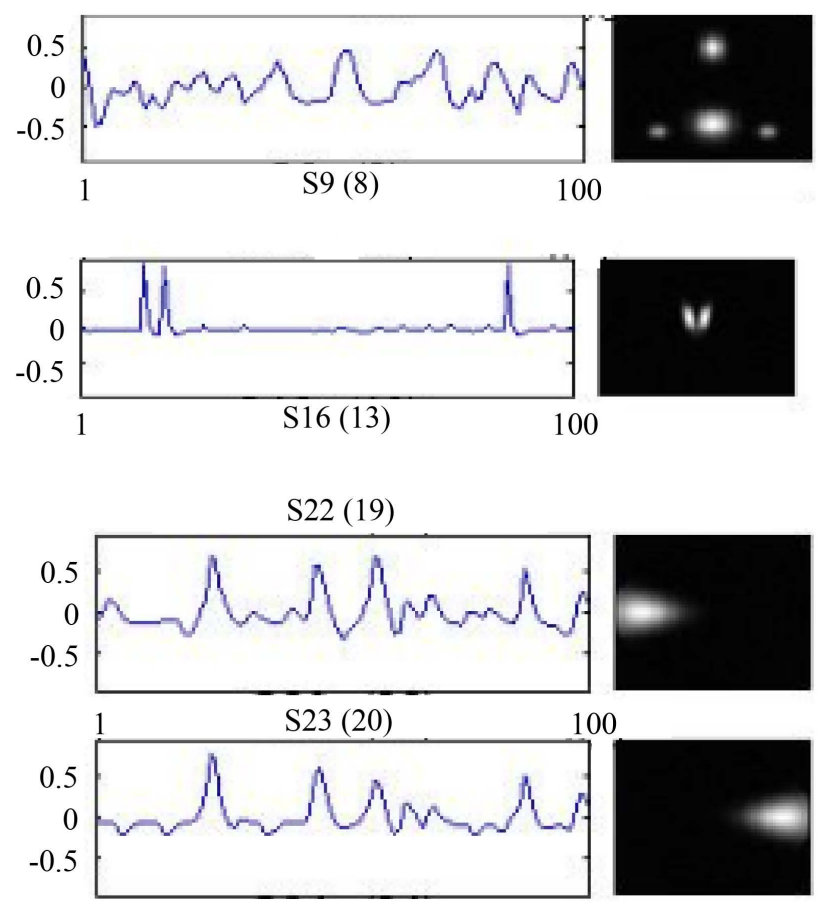

Figure 3. Simulated rsfMRI from simTB.

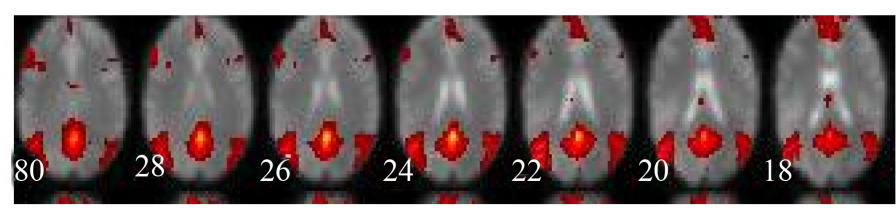

Figure 4. Spatial map of subject 3, Default mode network.
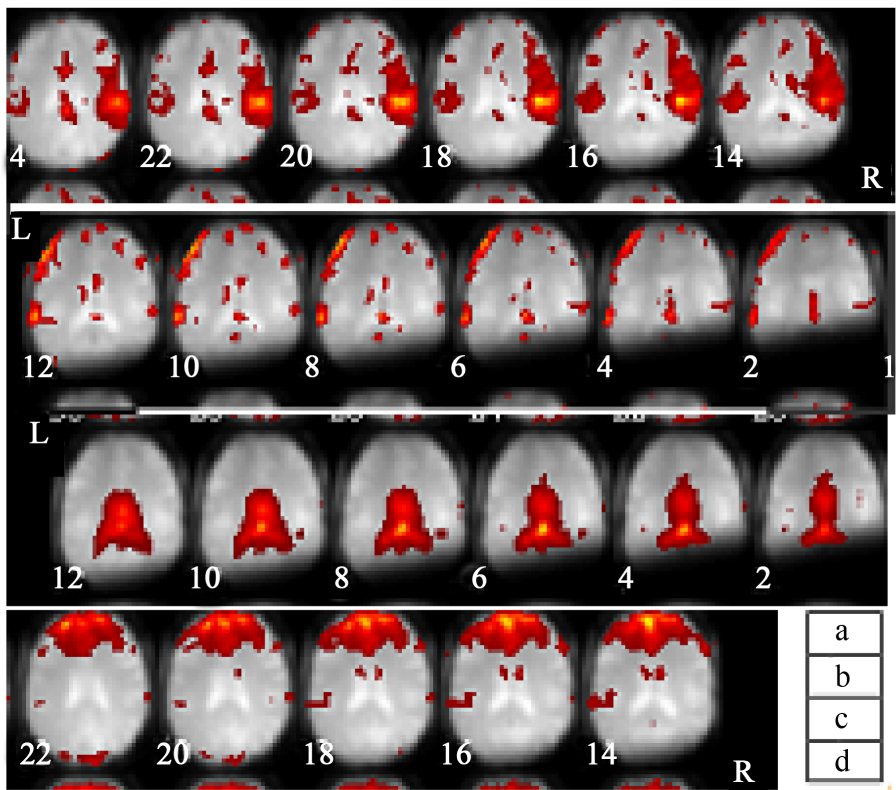

Figure 5. (a), (b) Subject 3 R \& L executive control network IC8, IC11, (c). Spatial map of independent component No. 15 of subject 3 showing BOLD response in sensory motor network. 


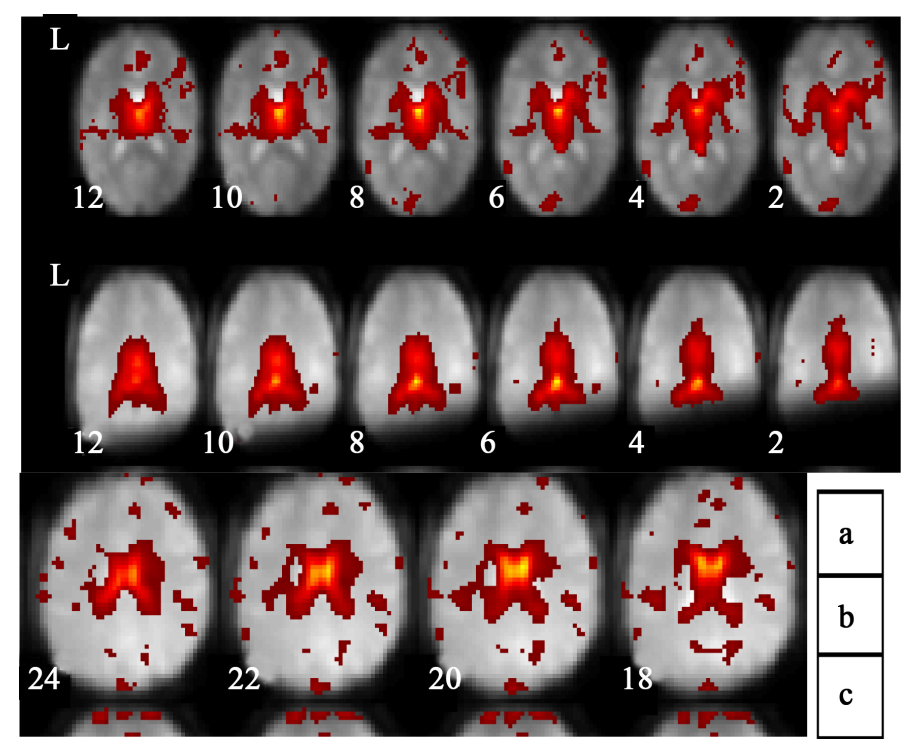

Figure 6. Sensory motor region of Healthy subject, (b) Spatial map of Independent Component No. 15 of BOLD response in Sensory motor network of Subject 3. (c) Spatial map of IC18, Sensory motor network.

of the subject. Nine healthy subjects whose mean age 70 years was compared with the functional activations of 3 abnormal shaky hand subjects, 9 acquisitions, of mean age 65 years.

\subsection{Two-Class Classifier}

The proposed ICA-PSO two-class classifier using cluster PCA is implemented for $128 \times 128 \times 23$ pixel $1.8 \times$ $1.8 \times 6 \mathrm{~mm}, 110$ volumes of 9 abnormal subjects and $64 \times 64 \times 40,3.44 \times 3.44 \times 3 \mathrm{~mm}, 110$ volumes of healthy controls. Each scan of rsfMRI in acquisition is Realigned, Normalized \& spatially smoothed. Thirty independent component's time series, for each scan is obtained using Group ICA as in Figure 7. The 30 Independent component time series with 19 scans of simulated and acquired shaky hand rsfMRI PCA are projected onto component space and two class clustering by cluster centroid and squared equilidian distance is performed.

Eighteen eigenvalues as given by PSO are considered. The accuracy of the classifier $87.5 \%$ for 30 trials, $82.36 \%$ specificity and precision $78 \%$, accounts to fine tune the number of IC chosen or the number of eigenvalues chosen for ICA-PSO two-class classifier.

\section{Discussions and Conclusions}

The main aim is to explore sensory motor cortex functional connectivity in aged subject's Shaky Hand (SH) patients and healthy controls (HCs) of SimTB simulated and acquired rsfMRI. The contribution lies in constructing a classifier by k-means on a 2D feature projection space and comparing simulated rsfMRI data with acquired data. The results inferred were compared in Figure 4 and Figure 7. The sensory motor region activation is high as compared to the healthy subject activations in both simulated rsfMRI and acquired data. The proposed algorithm ICA-PSO two-class classifier gives the classification of about $87.5 \%$ of the functional localization regions.

fMRI data were simulated by activating various brain regions involved in shaky hand persons in comparison with the healthy controls using SimTB, a MATLAB Toolbox. ICA automatically separated a time-series waveform representing neural activity associated large networks. The corresponding regions of activation identified by ICA overlapped roughly $92 \%$ with those identified by SimTB. For assessing region specific activation, SVM-GLM was used with IC finger print [10]. This work, ICA-PSO two-class classifier, is the first of its kind to use the IC time series eigenvalues for classification and comparison of simulated rest fMRI data with acquired data for shaky hand subjects. The future scope is to extract different statistical features such as region of interest, power spectral density to increase the classifier accuracy. 

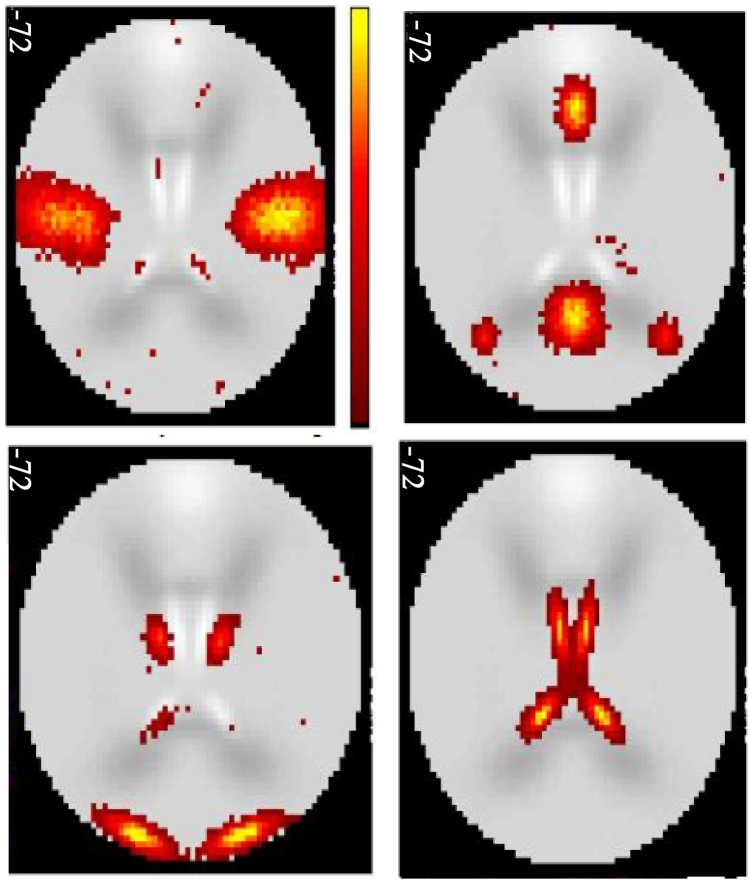

Figure 7. IC s of simulated HC rsfMRI using gift ICA.

\section{Acknowledgements}

The authors would like to show their gratitude to the Professor Dr Uma Maheshwara Rao, the head of Anesthesia and Academic Dean in NIMHANS, Dr Sri Ganesh, Associate Professor, Department of Neuroanaesthesia in NIMHANS, Bangalore, Dr Saini, Radiologist, NIMHANS for provided the Data.

\section{References}

[1] Jezzard, P., Matthews, P.M. and Smith, S.M. (2001) Functional MRI: An Introduction to Methods. Oxford University Press, Oxford.

[2] Cordes, D., Haughton, V.M., Arfanakis, K., Carew, J.D., Turski, P.A., Moritz, C.H., Quigley, M.A. and Meyerand, M.E. (2001) Frequencies Contributing to Functional Connectivity in the Cerebral Cortex in Resting-State Data. American Journal of Neuroradiology, 22, 1326-1333.

[3] Friston, K.J., Penny, W., Phillips, C., Kiebel, S., Hinton, G. and Ashburner, J. (2002) Classical and Bayesian Inference in Neuroimaging: Theory. NeuroImage, 16, 465-483. http://dx.doi.org/10.1006/nimg.2002.1090

[4] van de Ven, V.G., Formisano, E., Prvulovic, D., Roeder, C.H. and Linden, D.E.J. (2004) Functional Connectivity as Revealed by Spatial Independent Component Analysis of Fmri Measurements during Rest. Human Brain Mapping, 22, 165-178. http://dx.doi.org/10.1002/hbm.20022

[5] Calhoun, V.D., Adali, T., Stevens, M.C., Heil, K.A. and Pekar, J.J. (2005) Semi Blind ICA of fMRI: A Method of Utilizing Hypothesis Derived Time Courses in a Spatial ICA Analysis. NeuroImage, 25, 527-538.

http://dx.doi.org/10.1016/j.neuroimage.2004.12.012

[6] Greicius, M.D., Flores, B.H., Menon, V., Glover, G.H., Solvason, H.B., Kenna, H., Reiss, A.L. and Schatzberg, A.F. (2007) Resting-State Functional Connectivity in Major Depression: Abnormally Increased Contributions from Subgenual Cingulate Cortex and Thalamus. Biological Psychiatry, 62, 429-437. http://dx.doi.org/10.1016/j.biopsych.2006.09.020

[7] De Martino, F. (2007) Classification of fMRI Independent Components Using IC-Fingerprints and Support Vector Machine Classifiers. NeuroImage, 34, 177-194.

[8] Poli, R., Kennedy, J. and Blackwell, T. (2007) Particle Swarm Optimization: An Overview. Swarm Intelligence, 1, 3357. http://dx.doi.org/10.1007/s11721-007-0002-0

[9] Lindquist, M.A. (2008) The Statistical Analysis of fMRI Data. Statistical Science, 23, 439-464. http://dx.doi.org/10.1214/09-STS282 
[10] Wang, Z. (2009) A Hybrid SVM-GLM Approach for fMRI Data Analysis. NeuroImage, 46, 608-615. http://dx.doi.org/10.1016/j.neuroimage.2009.03.016

[11] Kelly Jr., R.E., et al. (2010) Visual Inspection of Independent Components: Defining a Procedure for Artifact Removal from fMRI Data. Journal of Neuroscience Methods, 189, 233-245. http://dx.doi.org/10.1016/j.jneumeth.2010.03.028

[12] Lee, K., Tak, S. and Ye, J.C. (2011) A Data-Driven Sparse GLM for fMRI Analysis Using Sparse Dictionary Learning with MDL Criterion. IEEE Transactions on Medical Imaging, 30, 1076-1089.

[13] Barbe, K., Van Maer, W. and Lauwers, L. (2011) Functional Magnetic Resonance Imaging: An Improved Short Record Signal Model. IEEE Transaction on Instrumentation and Measurements, 60, 1724-1731. http://dx.doi.org/10.1109/TIM.2010.2089150

[14] Demertzi, A., et al. (2014) Multiple fMRI System-Level Baseline Connectivity Is Disrupted in Patients with Consciousness Alterations. Cortex, 52, 35-46.

[15] Thaiyalnayaki, S.P., Rohini, A. and Uma Maheswari, O. (2015) Comparison of VAM Data Using SPM and ICA. International Journal of Applied Engineering Research, 10, 10817-10824.

\section{Submit or recommend next manuscript to SCIRP and we will provide best service for you:}

Accepting pre-submission inquiries through Email, Facebook, Linkedin, Twitter, etc A wide selection of journals (inclusive of 9 subjects, more than 200 journals)

Providing a 24-hour high-quality service

User-friendly online submission system

Fair and swift peer-review system

Efficient typesetting and proofreading procedure

Display of the result of downloads and visits, as well as the number of cited articles

Maximum dissemination of your research work

Submit your manuscript at: http://papersubmission.scirp.org/ 